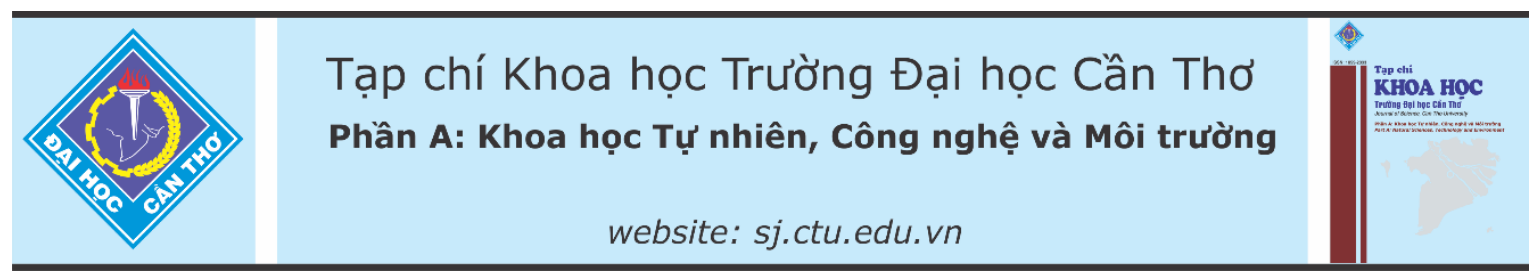

DOI:10.22144/ctu.jvn.2021.136

\title{
PHÂN TÍCH CÁC YẾU TỐ TÁC ĐỘNG ĐẾN HOẠT ĐỘNG CHUYỂN GIAO, ÚNG DỤNG, ĐỔI MỚI CÔNG NGHệ TẠI THÀNH PHỔ CẦN THƠ
}

\author{
Nguyễn Thắng Lợi, Nguyễn Hồng Phúc và Nguyễn Chí Ngôn* \\ Khoa Công nghệ, Truờng Đại hoc Cần Tho \\ *Người chịu trách nhiệm về bài viết: Nguyễn Chí Ngôn (email: ncngon@ctu.edu.vn)
}

\section{Thông tin chung:}

Ngày nhận bài: $24 / 08 / 2021$

Ngày nhận bài sưa: 23/09/2021

Ngày duyệt đăng: 29/10/2021

\section{Title:}

Analysis of factors affecting on technology transfer,

application, innovation in Can Tho city

\section{Tù khóa:}

Các yếu tố ảnh hwởng, chuyển giao công nghệ, doanh nghiẹp sản xuất, đổi mói công nghệ, úng dụng công nghệ

\section{Keywords:}

Influencing factors, production enterprises, technology application, technology innovation, technology transfer

\begin{abstract}
The purpose of the paper is to present an analysis of the Interpretive Structure Model and the Cross-Impact Matrix Multiplication Applied to Classification as a tool to classify the specific importance of the factors and their relationships. The hierarchical relationship between the factors helps to propose solutions to promote technology transfer, application and innovation in Can Tho city more closely and more effectively. These main factors are grouped in 04 large groups containing 14 sub-factors which are identified through survey results from 217 businesses in Can Tho city and consultations many experts on science and technology. The results showed that the cluster "Drivers" including the factors of Technological Proficiency, The business-specific nature, and Government strategy and policy has the strongest impact on this activity.
\end{abstract}

\section{TÓM TẮT}

Mục đỉch của bài báo là trình bày phân tích Mô hình cấu trúc diễn giải (Interpretive Structural Modeling-ISM) và phuoong pháp Phép nhân ma trận tác động chéo được áp dụng để phân loại (Cross-Impact Matrix Multiplication Applied to Classification-MICMAC) là một công cu phân loại mức độ quan trọng cu thể của các yếu tố và mối quan hệ thứ bậc giữa các yếu tố, giúp cho việc đề xuất các giải pháp thúc đẩy hoạt động chuyển giao, úng dụng, đổi mói công nghệ tại thành phố Cần Tho được chặt chẽ và đạt hiệu quả tốt hơn. Các yếu tố chính này bao gồm 04 nhóm yếu tố lớn chứa đựng 14 yếu tố phu được xác định thông qua các kết quả khảo sát 217 doanh nghiẹp trên địa bàn thành phố Cần Tho và tham vấn nhièu chuyên gia về khoa họ và công nghệ. Kết quả cho thấy các yếu tố trong cưm "Nhân tố định huoóng", bao gồm Khả năng thông thạo công nghệ, Tính chất đặc thù doanh nghiệp và Chiến lược và chính sách của Chính phủ có tác động mạnh mẽ nhất đến hoạt động này.

\section{GIỚI THIỆU}

Sự xuất hiện của cuộc cách mạng công nghiệp 4.0 đã tạo ra nhiều đột phá trong việc thay đổi nhận thức về công nghệ của thế giới cũng như của Việt Nam nói riêng. Ngày nay, đổi mới công nghệ đang là một vấn đề hết sức cấp bách, không chỉ giúp doanh nghiệp mà còn cả nền công nghệ của một đất nước có thể tồn tại và phát triển một các bền vững hơn. Đổi mới công nghệ được quan tâm đặc biệt hơn, theo đó, năm 2030, số lượng doanh nghiệp thực hiện đổi mới công nghệ tăng trung bình $20 \% /$ năm. $100 \%$ các doanh nghiệp sản xuất các sản phẩm chủ lực, sản phẩm trọng điểm hình thành tổ chức nghiên 
cứu và phát triển (Quyết định số $118 / \mathrm{Q} Đ-T T g$ ngày 25/01/2021 của Thủ tướng Chính phủ). Đổi mới công nghệ hướng đến mục tiêu nâng cao cả về số lượng lẫn chất lượng sản phẩm, và cả tính năng của sản phẩm với hao phí lao động trên một đơn vị sản phẩm thấp hơn, từ đó có khả năng làm hạ giá thành sản phẩm, đáp ứng yêu cầu ngày càng khắt khe của khách hàng trên thị trường (Nguyễn Thị Thơm, 2020). Tuy nhiên, hiện nay tình hình đổi mới công nghệ của doanh nghiệp đang diễn ra còn chậm mặc dù thực tế đã có nhiều doanh nghiệp Việt Nam đã bắt đầu thay thế sản phẩm và dịch vụ của mình bằng những sản phẩm và dịch vụ mới, cải tiến, sáng tạo hơn.

Đồng bằng sông Cửu Long (ĐBSCL) là một vùng kinh tế trọng điểm nằm ở phía Nam của Việt Nam cũng không nằm ngoài xu thế đó. Với vai trò là đầu tàu kinh tế của khu vực ĐBSCL, thành phố Cần Thơ (TP. Cần Thơ) ngày càng thể hiện vai trò quan trọng hơn trong phát triển công nghiệp, nông nghiệp và dịch vụ của toàn vùng. Trong vòng 5 năm qua (2016-2020), giá trị sản xuất công nghiệp của TP. Cần Thơ đóng góp khoảng 15,2\% trong cơ cấu công nghiệp của toàn vùng và chiếm tới $44,4 \%$ tổng giá trị sản xuất công nghiệp toàn vùng kinh tế trọng điểm ĐBSCL (An Giang, Cà Mau, Cần Thơ, Kiên Giang) (Ngọc Thảo, 2021). Theo giá trị đóng góp của từng nhóm ngành, tính đến nay TP. Cần Thơ có $5 / 9$ nhóm ngành có tính dẫn dắt công nghiệp vùng ĐBSCL như sản xuất, chế biến thực phẩm, đồ uống (chiếm 46,2\%); hóa chất, hóa dược (chiếm 54,9\%); cơ khí, sản xuất kim loại (chiếm 57,4\%); dệt may, da giày (chiếm 41,6\%)... Riêng về giá trị công nghiệp, ngành chế biến thủy sản Cần Thơ đạt kim ngạch xuât khẩu khoảng 570 triệu USD/năm, chiếm từ $45-50 \%$ tổng giá trị xuất khẩu và ngành xay xát, chế biến gạo đạt công suất bình quân từ 4,2- 4,3 triệu tấn/năm (Ngọc Thảo, 2021). Bên cạnh đó, nông nghiệp công nghệ cao và dịch vụ cũng là các thế mạnh khác của thành phố. Mặc dù có những đóng góp quan trọng cho sự phát triển kinh tế của ĐBSCL nói chung và địa bàn thành phố nói riêng, việc quản lý về khoa học và công nghệ của Cần Thơ còn gặp rất nhiều thách thức, đặc biệt là các yêu cầu ngày càng khắt khe về khép kín quy trình sản xuất để tạo ra những sản phẩm truy xuất nguồn gốc từ đầu vào đến đầu ra cũng như những khó khăn khủng hoảng do ảnh hưởng từ dịch bệnh.

Trong quản lý công nghệ, đặc biệt là quản lý công nghệ trong tương lai, quá trình xác định và phân tích các yếu tố quyết định sự phát triển trong tương lai của công nghệ đóng một vai trò quan trọng (Halicka, 2016). Các yếu tố này có thể thuộc loại công nghệ, kinh tế, xã hội, luật pháp, đạo đức hoặc môi trường. Sự đa dạng của các yếu tố này thường đòi hỏi phân tích chuyên sâu với mục đích là tổ chức các yếu tố và xác định các yếu tố đóng vai trò quan trọng. Sự tập trung của các hoạt động trong tương lai và chiến lược phát triển vào các yếu tố quan trọng của sự phát triển công nghệ có thể đóng góp vào thành công cuối cùng. Trong bối cảnh quản lý triển vọng phát triển công nghệ, việc lựa chọn các yếu tố chính có thể tạo cơ sở cho việc xây dựng các kịch bản phát triển mong muốn của đối tượng hoặc hệ thống. Một cách để tạo ra các kịch bản do Klooster and Asselt (2006) đề xuất là kỹ thuật trục kịch bản, áp đặt việc xác định hai yếu tố chính quyết định hoạt động của một hệ thống cụ thể. Các nghiên cứu về việc sử dụng phương pháp kịch bản cho mục đích nghiên cứu tầm nhìn xa do Nazarko and Kononiuk (2013) thực hiện đã khẳng định tính khả dụng và phổ biến cao của nó.

Bài báo này trình bày phân tích Mô hình cấu trúc diễn giải (Interpretive Structural Modeling-ISM) kết hợp với phương pháp Phép nhân ma trận tác động chéo được áp dụng để phân loại (Cross-Impact Matrix Multiplication Applied to Classification MICMAC) như một công cụ xác định và phân loại các yếu tố ảnh hưởng đến việc đề xuất các giải pháp thúc đẩy hoạt động chuyển giao, ứng dụng, đổi mới công nghệ. Bên cạnh đó, các ý nghĩa thực tiễn của phương pháp được trình bày dưới dạng một nghiên cứu điển hình của đề tài nghiên cứu khoa học xã hội và nhân văn cấp thành phố có tên "Nghiên cứu giải pháp thúc đẩy hoạt động chuyển giao, ứng dụng, đổi mới công nghệ tại thành phố Cần Thơ" với mục đích cập nhật thường xuyên và đề xuất các giải pháp kịp thời thúc đẩy hoạt động chuyển giao, ứng dụng, đồi mới công nghệ trên địa bàn theo hướng ổn định và bền vững.

\section{PHƯƠNG PHÁP NGHIÊN CÚU}

\subsection{Lược khảo tài liệu và mô hình nghiên cứu}

Về đổi mới và chuyển giao công nghệ, luật Chuyển giao công nghệ Việt Nam năm 2017 đã định nghĩa như sau:

- Đổi mới công nghệ là hoạt động thay thế một phần hoặc toàn bộ công nghệ đang sử dụng bằng một phần hoặc toàn bộ công nghệ khác nhằm nâng cao năng suất, chất lượng và khả năng cạnh tranh của sản phẩm.

- Chuyển giao công nghệ là chuyển nhượng quyền sở hữu công nghệ hoặc chuyển giao quyền sử dụng công nghệ từ bên có quyền chuyển giao công nghệ sang bên nhận công nghệ. 
Theo Cancino et al. (2018), đổi mới công nghệ được coi là phương tiện để tối ưu hóa việc sử dụng hiệu quả các nguồn lực quan trọng trong các hệ thống kinh tế xã hội-sinh học.

Lược khảo qua các giáo trình về Quản trị Công nghệ được dịch thuật từ tài liệu Morel-Guimaraes et al. (2005), sự tương tác giữa công nghệ và môi trường xung quanh bao gồm 7 nhóm yếu tố (được thống kê trong Hình 1):

- Dân số: Một công nghệ có thể tác động đến tốc độ tăng trưởng dân sô,, tuổi thọ, cơ cấu dân số theo các tiêu chí khác nhau, trình độ học vấn và các đặc điểm về lao động (mức thất nghiệp và cơ cấu lao động).

- Kinh tế: Các chỉ tiêu phản ánh yếu tố này có thể là tính khả thi về kinh tế (chi phí - lợi ích), cải thiện năng suất (vốn và các nguồn lực khác), tiềm năng thị trường (qui mô, độ co giãn), tốc độ tăng trưởng và cơ cấu kinh tế.

- Môi truò̀ng: Các chỉ tiêu phản ánh yếu tố này bao gồm môi trường vật chất (không khí, nước, chất thải rắn và đất đai), khí tượng và thủy văn, điều kiện sống (mức độ thuận tiện và tiếng ồn), cuộc sống (độ an toàn và sức khoẻ), môi sinh và hệ sinh thái.
- Đầu vào: Một công nghệ có thể tác động đến mức độ dồi dào của nguyên vật liệu và năng lượng, tài chính và nguồn nhân lực có tay nghề.

- Công nghệ: Các chỉ tiêu liên quan đến khía cạnh kỹ thuật như năng lực, độ tin cậy và hiệu quả; các phương án lựa chọn công nghệ như độ linh hoạt và quy mô; mức độ phát triển của hạ tầng như sự hỗ trợ và dịch vụ, năng lực sử dụng vận hành, công nghệ cung cấp đầu vào và công nghệ sử dụng đầu ra.

- Văn hoá - xã hội: Thuộc nhóm yếu tố này có yếu tố như tôn giáo, hành vi tiêu dùng, phong tục tập quán và chân giá trị của xã hội.

- Chính trị - pháp lý: Các yếu tố chính trị pháp lý bao gồm đảng cầm quyền, hệ thống chính trị, hệ thống pháp luật và quan hệ quốc tế. Trên thế giới, Guo et al. (2018) tổng hợp được rằng sự hỗ trợ của Chính phủ thông qua quỹ nghiên cứu và phát triển ảnh hưởng tích cực đến đổi mới tại các doanh nghiệp Trung Quốc. Jugend et al. (2018) nghiên cứu tại công ty Brazil cũng kết luận sự hỗ trợ của Chính phủ rất quan trọng trong việc áp dụng đổi mới. Không chỉ hỗ trợ về mặt tài chính, việc hỗ trợ về khía cạnh phi tài chính cũng rất quan trọng.

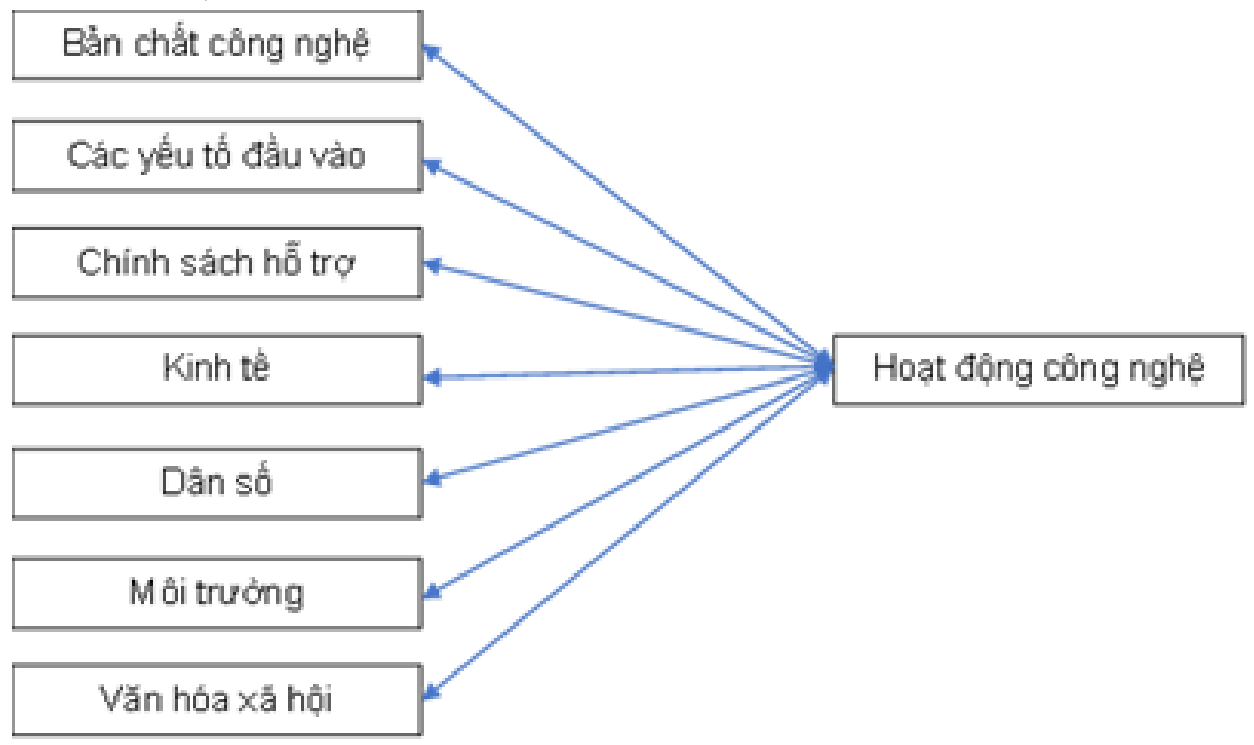

Hình 1. Các yếu tố tác động đến hoạt động về công nghệ

Nguồn: Morel-Guimaraes et al. (2005)

Danh mục các yếu tố thuộc từng nhóm có thể còn dài hơn nữa, phụ thuộc vào từng công nghệ cụ thể. Các yếu tố phụ này có thể thay đổi theo thời gian vì vậy mức độ tác động của chúng cũng thay đồi. Điều này đòi hỏi hoạt động về công nghệ cũng mang tính động.
Nghiên cứu về các yếu tố ảnh hưởng đến hoạt động chuyển giao công nghệ quốc tế trong và ngoài nước đã được triển khai xem xét như: phân tích nhân tố ảnh hưởng đại diện cho mức độ tương quan, quy trình phân tích thứ bậc, phương pháp đánh giá đa nhân tố và nhân tố chính, phân tích nhân tố, phân 
tích thành phần chính, phương pháp đánh giá toàn diện mờ,... Nhìn chung, phương pháp nghiên cứu các yếu tố ảnh hưởng là thiết lập hệ thống chỉ số đánh giá sau khi thu thập thông tin và xác định trọng số. Trong khi quyết định về trọng số là một chút chủ quan và xác suất vì thời gian khác nhau và khu vực khác nhau, điều này sẽ ảnh hưởng đến độ chính xác của phân tích thực nghiệm. Một số chỉ số hầu như không được đo lường theo dữ liệu hiện có. Mô hình ISM có thể loại bỏ tính chủ quan từ người định giá và tránh đánh giá không đầy đủ do thiếu dữ liệu (Yang et al., 2014).

Giai doạn 1

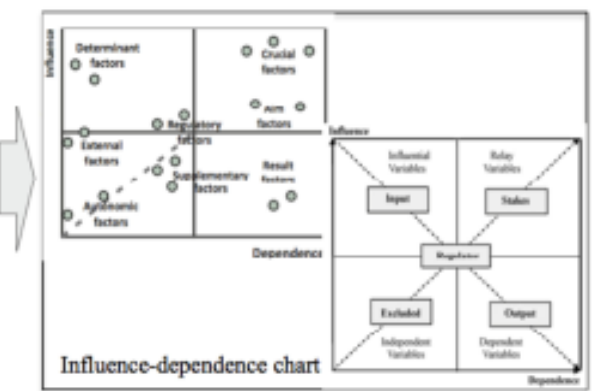

Nghiên cứu này phát triển một cách tiếp cận phân tích tích hợp để mô hình hóa các yếu tố tác động đến hoạt động chuyển giao, ứng dụng, đổi mới công nghệ tại thành phố Cần Thơ. bằng cách sử dụng phương pháp ISM và MICMAC để phân loại nhóm yếu tố. Trong cách tiếp cận này, trước hết các yếu tố tác động đến hoạt động chuyển giao, ứng dụng, đổi mới công nghệ tại thành phố Cần Thơ và các tiêu chí phụ trong mỗi yếu tố đó được xác định, sau đó ISM và MICMAC được triển khai. Theo Ahmed et al. (2016) và Ejdys et al. (2016), quá trình cụ thể dựa trên ba giai đoạn cơ bản (Hình 2).

\footnotetext{
Xác định các yếu tố có ảnh hưởng đến sự kiện đã cho
}

Giai đoạn 2

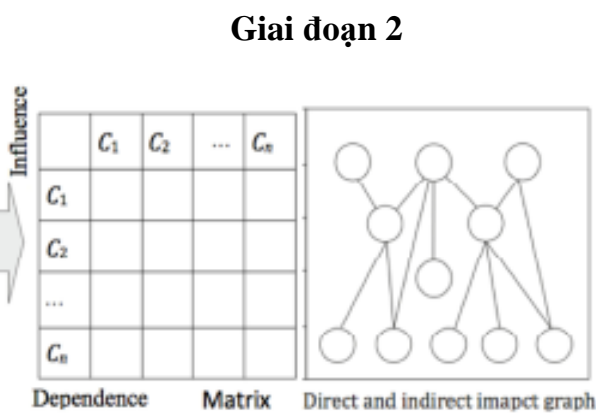

\section{Hình 2. Sơ đồ quy trình nghiên cứu}

\section{Giai đoạn 3}

\section{Nguồn: Ejdys et al. (2016).}

\subsection{Phương pháp mô hình hóa cấu trúc và phương pháp nhân ma trận tác động chéo áp dụng cho phân loại}

\subsubsection{Phuong pháp ISM}

Phương pháp ISM là một công cụ cho phép phát hiện mối quan hệ và ảnh hưởng lẫn nhau giữa các trình điều khiển của hệ thống được nghiên cứu (Warfield, 1973). Uuu điểm của phân tích cấu trúc là khả năng xác định mối quan hệ giữa các biến số mà ảnh hưởng lẫn nhau của chúng không rõ ràng và có thể không được các chuyên gia trong lĩnh vực này công nhận. Bằng cách phân tích sự phụ thuộc giữa các yếu tố bề ngoài không liên quan, phương pháp phân tích cấu trúc cho phép các nhà nghiên cứu mô tả tác động lẫn nhau, cũng như phản ứng; dựa trên những phản ứng này có thể phân biệt được biến nào là cốt yếu (Godet \& Durance, 2008).

Phương pháp ISM tạo ra một quy trình thực hiện so sánh các cặp yếu tố để biến đổi một vấn đề phức tạp thành một mô hình cấu trúc dễ hiểu hơn (Warfield, 1973). Quy trình thực hiện ISM gồm 6 bước cơ bản.

- Bước 1: Nhận diện và xác định các yếu tố dùng để đánh giá, lựa chọn nhà cung ứng được xác định dựa trên khảo sát thực tế doanh nghiệp hoặc các nghiên cứu trước đó.

- Bước 2: Xây dựng ma trận tương tác cấu trúc dựa trên mối quan hệ giữa các tiêu chí.

+ Bản câu hỏi được thiết kế để thu thập ý kiến của chuyên gia về mối quan hệ giữa từng tiêu chí với các tiêu chí còn lại.

+ Mối quan hệ giữa yếu tố $\mathrm{i}$ và yếu tố $\mathrm{j}$ được thể hiện như sau:

V: Tiêu chí i sẽ giúp đạt được/ dẫn đến tiêu chí j.

A: Tiêu chí $\mathrm{j}$ sẽ giúp đạt được/ dẫn đến tiêu chí i.

$X$ : Tiêu chí $\mathrm{i}$ và $\mathrm{j}$ liên quan lẫn nhau.

O: Tiêu chí i và j không liên quan đến nhau.

- Bước 3: Xây dựng ma trận tiếp cận ban đầu dựa trên ma trận tương tác cấu trúc Dữ liệu trong ma trận tương tác cấu trúc được chuyển thành mã nhị phân như sau: 
+ Nếu giá trị $(i, j)$ trong ma trận ban đầu là $\mathrm{V}$ thì giá trị $(\mathrm{i}, \mathrm{j})$ trong ma trận tiếp cận sẽ chuyển thành 1 . Đồng thời, giá trị $(\mathrm{j}, \mathrm{i})$ sẽ chuyền thành 0 .

+ Nếu giá trị $(i, j)$ trong ma trận ban đầu là A thì giá trị $(\mathrm{i}, \mathrm{j})$ trong ma trận tiếp cận sẽ chuyển thành 0 . Đồng thời, giá trị $(\mathrm{j}, \mathrm{i})$ sẽ chuyển thành 1 .

+ Nếu giá trị (i,j) trong ma trận ban đầu là $\mathrm{X}$ thì cả 2 giá trị $(\mathrm{i}, \mathrm{j})$ và $(\mathrm{j}, \mathrm{i})$ trong ma trận tiếp cận đều chuyển thành 1 .

+ Nếu giá trị $(i, j)$ trong ma trận ban đầu là $\mathrm{O}$ thì cả 2 giá trị $(\mathrm{i}, \mathrm{j})$ và $(\mathrm{j}, \mathrm{i})$ trong ma trận tiếp cận đều chuyển thành 0 .

- Bước 4: Xây dựng ma trận tiếp cận cuối cùng. Ma trận tiếp cận cuối cùng được xây dựng bằng cách xem xét mối quan hệ của các tiêu chí trong bảng theo nguyên tắc: Nếu tiêu chí $\mathrm{i}$ có liên quan đến tiêu chí $\mathrm{j}$ và tiêu chí $\mathrm{j}$ có liên quan đến tiêu chí $\mathrm{k}$ thì tiêu chí $\mathrm{i}$ cũng sẽ liên quan đến tiêu chí $\mathrm{k}$. Khi đó, giá trị trong ô $(\mathrm{i}, \mathrm{k})$ sẽ được chuyển từ 0 sang 1.

- Bước 5: Phân cấp các yếu tố Từ ma trận tiếp cận cuối cùng, xác định các yếu tố quan hệ theo hàng và quan hệ theo cột theo nguyên tắc:

+ Xét theo từng tiêu chí, các tiêu chí nào có mối quan hệ với nhau được liệt kê tương ứng.

+ Sau đó, xác định các tiêu chí giao nhau trong tất cả tiêu chí liệt kê.

+ Cuối cùng, tiêu chí nào có tập hợp các mối quan hệ theo hàng và giao điểm giữa hàng và cột giống nhau được chia bậc (từ I đến VIII) và loại bỏ ở quá trình xếp bậc tiếp theo.

- Bước 6: Hình thành mô hình ISM dựa trên kết quả phân cấp các yếu tố, sơ đồ cấu trúc được hình thành. Sơ đồ cấu trúc thể hiện khá toàn diện mối quan hệ giữa các tiêu chí, hỗ trợ cho quá trình đánh giá mức độ quan trọng của từng tiêu chí ở giai đoạn tiếp theo.

\subsubsection{Phuoong pháp MICMAC}

Sử dụng các kết quả từ hương pháp ISM, các giá trị hàng và cột dựa trên ma trận tiếp cận cuối cùng được sử dụng cho việc phân chia các yếu tố vào các cụm theo phương pháp nhân ma trận tác động chéo áp dụng cho phân loại (MICMAC). Trong đó, tổng các giá trị theo hàng được gọi là mức độ định hướng/độc lập (Driving Power-DrP) và tổng các giá trị theo cột được gọi là mức độ phụ thuộc (Dependent Power-DP).

Các yếu tố được chia thành 4 cụm, cụ thể như sau:

- Cụm các yếu tố tự quản: Các yếu tố có DP và DrP thấp.

- Cụm các yếu tố phụ thuộc: Cụm các yếu tố có $\mathrm{DP}$ cao và $\mathrm{DrP}$ thấp.

- Cụm các yếu tố liên kết: Cụm các yếu tố có $\mathrm{DP}$ và $\mathrm{DrP}$ cao.

- Cụm các yếu tố định hướng: Cụm các yếu tố có $\mathrm{DP}$ thấp và $\mathrm{DrP}$ cao.

\subsubsection{Phurong pháp xác định số đơn vị mẫu điều tra, khảo sát}

Đối với điều tra khảo sát doanh nghiệp, đề tài sử dụng phương pháp chọn mẫu ngẫu nhiên phân tầng theo lĩnh vực, áp dụng công thức Slovin để xác định tổng số quan sát mẫu nhằm đảm bảo tính đại diện của nghiên cứu. Theo Slovin (1984), cỡ mẫu được xác định theo công thức sau:

$$
n=\frac{N}{1+N \times e^{2}}
$$

Trong đó:

- N: tổng số doanh nghiệp đang hoạt động tại TP. Cần Thơ

- n: số doanh nghiệp đại diện

- e: sai số cho phép (chọn e $=9 \%$, sai số cho phép $<10 \%$ )

Tính cỡ mẫu doanh nghiệp sẽ thu thập thông tin về chuyển giao, ứng dụng, đồi mới công nghệ theo công thức của Slovin (1984). Chọn mức sai số cho phép là $9 \%$ thì số doanh nghiệp cần điều tra tối thiểu là 210 doanh nghiệp. Số lượng doanh nghiệp theo từng lĩnh vực nghiên cứu đã được thu thập như thông tin hiển thị ở Bảng 1. 
Bảng 1. Số lượng doanh nghiệp theo các lĩnh vực nông nghiệp, công nghiệp và dịch vụ tại TP. Cần Tho

\begin{tabular}{|c|c|c|}
\hline Stt & Lĩnh vực & $\begin{array}{l}\text { Số doanh nghiệp } \\
\text { điều tra, khảo sát }\end{array}$ \\
\hline 1 & $\begin{array}{l}\text { Nông nghiệp } \\
\text { (gồm các doanh nghiệp hiện đang hoạt động có mã ngành đăng ký } \\
\text { thuộc mã ngành A: Nông nghiệp, lâm nghiệp, thủy sản) }\end{array}$ & 22 \\
\hline 2 & $\begin{array}{l}\text { Công nghiệp } \\
\text { (gồm các doanh nghiệp hiện đang hoạt động có mã ngành đăng ký } \\
\text { thuộc mã ngành } \mathbf{B} \text { : Khai khoáng; C: Công nghiệp chế biến, chế tạo; } \mathbf{D} \text { : } \\
\text { Sản xuất và phân phối điện, khí đốt, nước nóng, hơi nước và điều hoà } \\
\text { không khí; E: Cung cấp nước; hoạt động quản lý và xử lý rác thải, nước } \\
\text { thải; } \mathbf{F} \text { : Xây dựng) }\end{array}$ & 110 \\
\hline 3 & 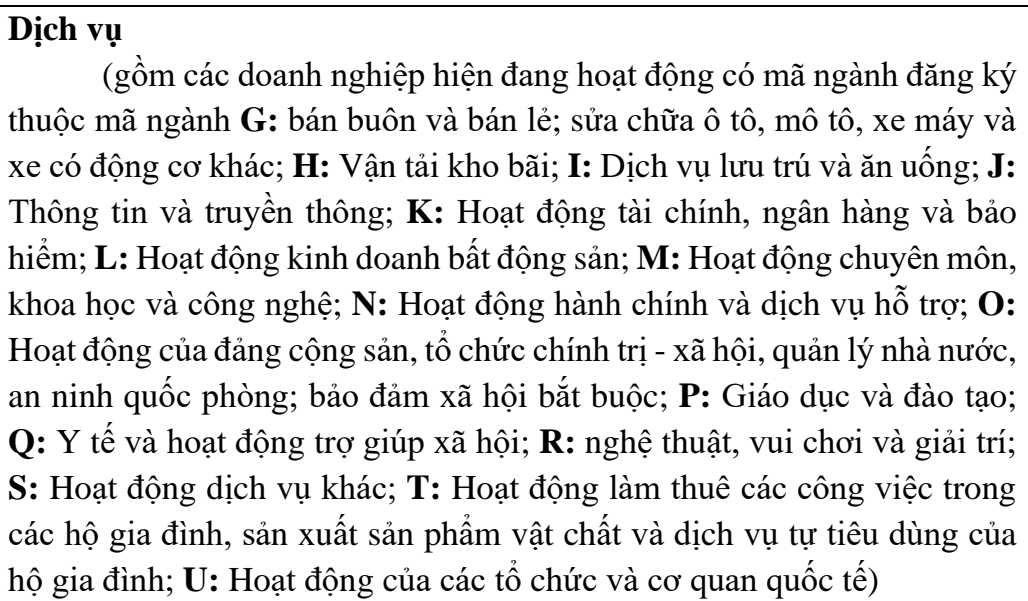 & 85 \\
\hline & Tổng số (doanh nghiệp) & 217 \\
\hline
\end{tabular}

Nguồn: Theo điều tra thục tế của Cục thống kê TP. Cần Tho (2019 - 2020).

Bên cạnh đó, TP. Cần Thơ có 73 tổ chức có hoạt động khoa học và công nghệ trên địa bàn thành phố (theo báo cáo Thống kê Khoa học và Công nghệ, Cục thống kê TP. Cần Thơ, 2019).

Áp dụng công thức của Slovin (1984) để tính cỡ mẫu khảo sát thu thập thông tin về chuyển giao, ứng dụng, đổi mới công nghệ tại các cơ quan, tổ chức này. Nếu mức sai số cho phép được chọn là $9 \%$ thì số cơ quan, tổ chức có hoạt động khoa học và công nghệ cần khảo sát tối thiểu là 46 đơn vị. Mẫu đơn vị khảo sát được chọn theo phương pháp cắt ngang.
Trên cơ sở 46 cơ quan, tổ chức có hoạt động khoa học và công nghệ khảo sát, công thức của Slovin (1984) được áp dụng tiếp tục, với mức sai số cho phép là $9 \%, 33$ đơn vị được chọn để phỏng vấn sâu các chuyên gia, lãnh đạo của các đơn vị này về chuyển giao, ứng dụng, đổi mới công nghệ. Mẫu phỏng vấn sâu được chọn theo phương pháp cắt ngang.

- Phương pháp tổng hợp thông tin:

Khung nghiên cứu chi tiết của bài báo được hiển thị ở Hình 3. 


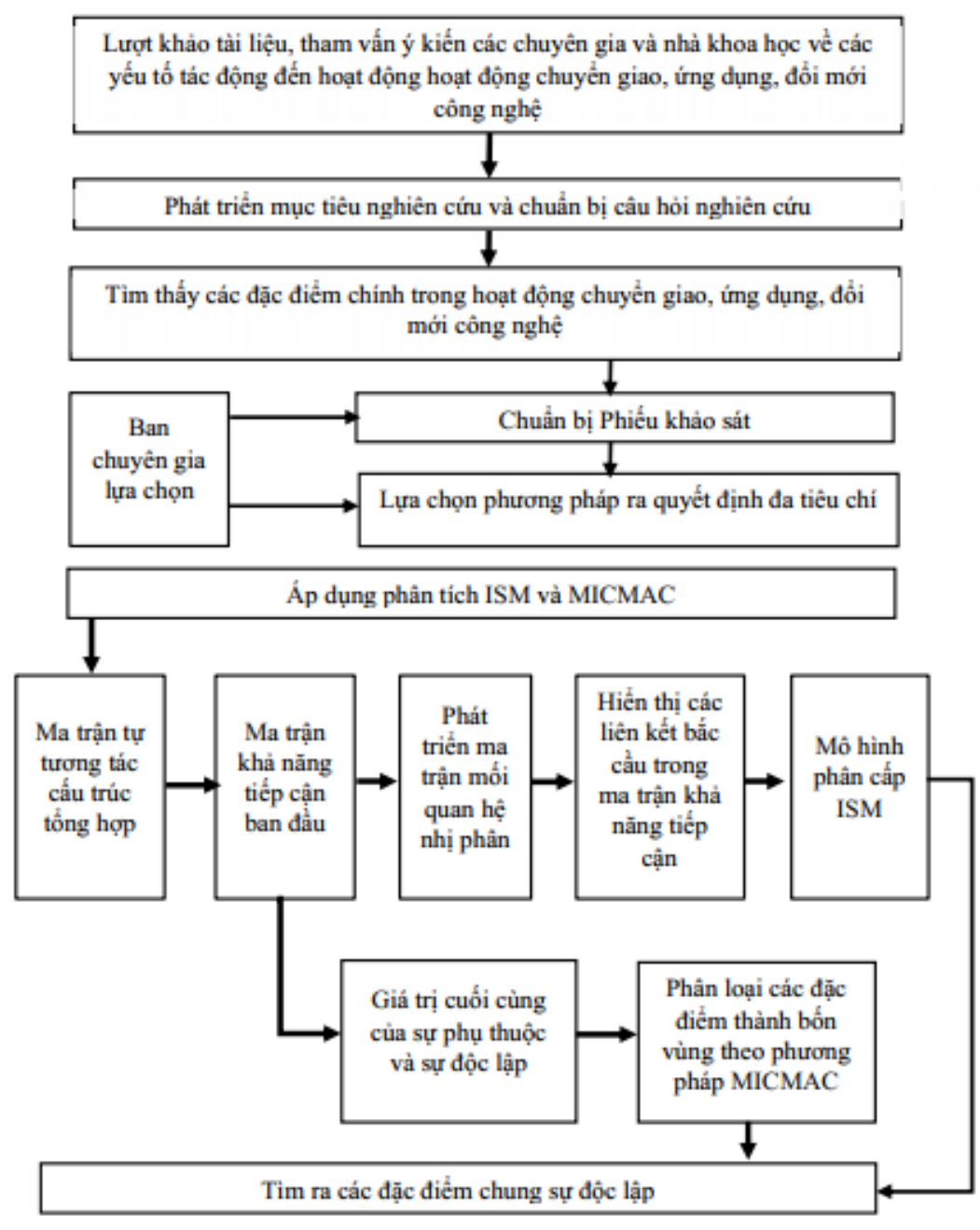

Hình 3. Khung nghiên cứu

Từ việc thừa hưởng các kết quả từ MorelGuimaraes et al. (2005), nhóm nghiên cứu đã tiến hành tham vấn ý kiến chuyên gia và xem xét tình hình thực tế của địa phương là TP. Cần Thơ để xác định các yếu tố được xem là tạo ra nhiều tác động nhất đến hoạt động về công nghệ trên địa bàn. Do đó, một mô hình nghiên cứu gồm các yếu tố được đề xuất và thể hiện trong Hình 4.

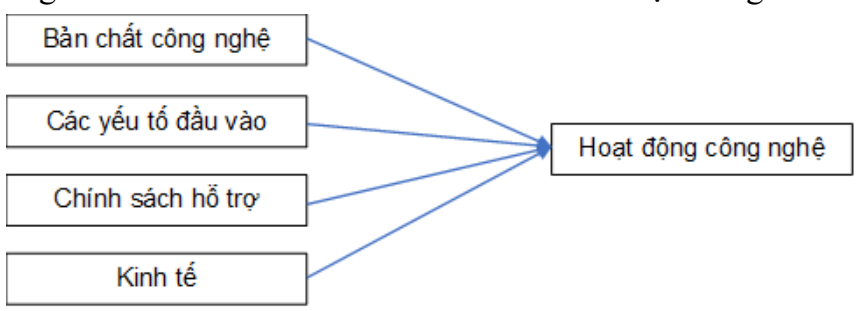

\section{Hình 4. Mô hình nghiên cứu về các yếu tố tác động đến hoạt động về công nghệ}

\section{KẾT QUẢ VÀ THẢO LUẬN}

Sử dụng phương pháp ISM kết hợp với MICMAC để tiến hành xem xét mức độ tác động của các yếu tố với các bước cụ thể như sau:
- Bước 1: Nhận diện và xác định các yếu tố

Các yếu tố phụ đính kèm được trình bày dựa vào khung lý thuyết. Số lượng lớn các phiếu khảo sát được thu lại tập trung giải đáp các vấn đề mà doanh 
nghiệp và các chuyên gia tổ chức quan tâm bao gồm:

(1) Bản chất công nghệ; (2) Các yếu tố đầu vào, (3)
Chính sách hỗ trợ và (4) Kinh tế. Các yếu tố phụ thuộc về 4 nhóm này được hiển thị ở Bảng 2.

Bảng 2. Các yếu tố được quan tâm nhất đối với hoạt động chuyển giao, ứng dụng, đổi mới công nghệ của TP. Cần Tho

\begin{tabular}{llc}
\hline Yếu tố chính & Yếu tố trực thuộc/phụ & Ký hiệu \\
\hline \multirow{3}{*}{ Bản chất công nghệ } & Khả năng thông thạo công nghệ & F1 \\
& Mức độ phức tạp của công nghệ & F2 \\
& Tính chất đặc thù doanh nghiệp & F3 \\
\hline \multirow{3}{*}{ Các yếu tố đầu vào } & Tài chính của doanh nghiệp & F4 \\
& Nguồn nhân lực & F5 \\
& Tiêu hao năng lượng & F6 \\
\hline \multirow{3}{*}{ Chính sách hỗ trợ } & Chiến lược và chính sách của Chính phủ & F7 \\
& Cơ chế chuyển giao & F8 \\
& Khả năng tiếp nhận công nghệ quốc tế & F9 \\
& Mức độ ưu tiên của dự án & F10 \\
\hline \multirow{3}{*}{ Kinh tế } & Thị trường công nghệ không đầy đủ và thông tin không chắc chắn & F11 \\
& Sự thay đổi của cơ cấu kinh tế & F12 \\
& Vốn sản xuất & F13 \\
& Cạnh tranh tiếp thị sản phẩm đầu ra & F14 \\
\hline
\end{tabular}

Về mức độ đóng góp thực tế của các yếu tố này đến hoạt động chuyển giao công nghệ phục vụ phát triển kinh tế xã hội của TP. Cần Thơ, hoạt động trong lĩnh vực nông nghiệp, công nghiệp và dịch vụ được phân tích và tổng hợp từ quá trình khảo sát các chuyên gia và khảo sát chuyên sâu, kết quả được thể hiện trong Hình 3.

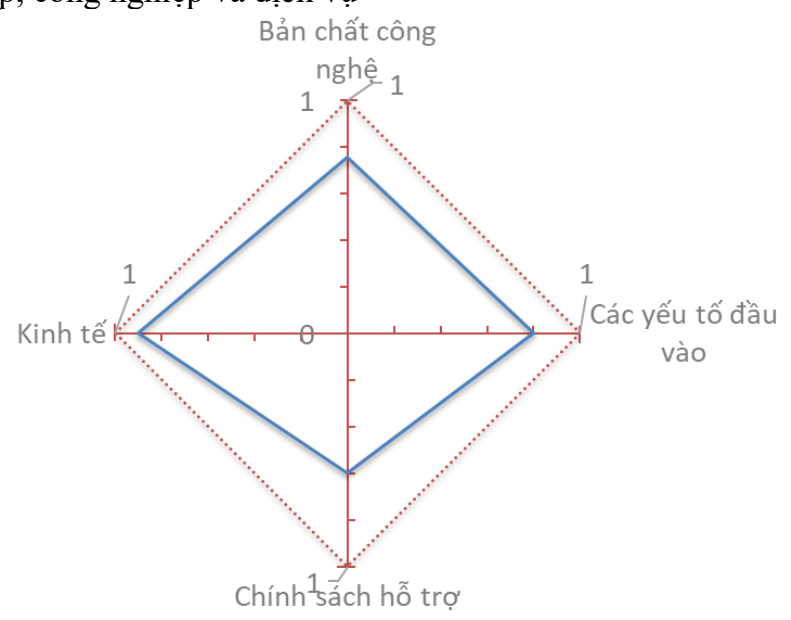

\section{Hình 3. Hiện trạng công nghệ của các doanh nghiệp trên địa bàn thành phố Cần Thơ}

Nguồn: Tổng hợp tù Cuc thống kê TP. Cần Tho (2020) và phỏng vấn chuyên sâu chuyên gia (2020)

Hình 3 cho thấy hiện trạng công nghệ của các doanh nghiệp so với mức mong muốn của doanh nghiệp trong việc phát triển năng lực công nghệ của họ còn một khoảng cách, xét về các khía cạnh kinh tế, bản chất công nghệ, các yếu tố đầu và chính sách hỗ trợ. Kết quả này cho thấy hoạt động quản lý công nghệ trong doanh nghiệp từ các cấp quản lý nhà nước vẫn còn nhiều khó khăn và khúc mắc vẫn chưa thể giải quyết một cách triệt để. Do đó, hoạt động xác định hiện trạng và mức độ quan trọng của tác yếu tố tác động là một vấn đề cấp thiết. Kết quả từ quá trình này có thể đóng góp các yếu tố mấu chốt để mang lại tính hiệu quả cao hơn trong việc xác định và thực thi các giải pháp liên quan.

- Bước 2: Xây dựng ma trận tương tác cấu trúc dựa trên mối quan hệ giữa các yếu tố 
Các nội dung của phiếu khảo sát ý kiến đối với các chuyên gia và nội dung phỏng vấn chuyên sâu được tập trung so sánh từng cặp nhân tố theo quy tắc của phương pháp ISM. Kết quả từ quá trình khảo sát về các giá trị về mức độ tương tác được tổng hợp lại ở Bảng 3.

Bảng 3. Ma trận tương tác cấu trúc dựa trên mối quan hệ giữa các yếu tố

\begin{tabular}{|c|c|c|c|c|c|c|c|c|c|c|c|c|c|c|}
\hline & F1 & $\overline{F 2}$ & F3 & F4 & F5 & F6 & F7 & F8 & F9 & F10 & F11 & F12 & F13 & F14 \\
\hline F1 & 1 & $\mathrm{~A}$ & $\mathrm{~V}$ & $\mathrm{O}$ & $\mathrm{V}$ & $\mathrm{V}$ & $\mathrm{O}$ & $\mathrm{O}$ & $\mathrm{V}$ & $\mathrm{V}$ & $\bar{V}$ & $\mathrm{~V}$ & $\mathrm{~V}$ & $\mathrm{~V}$ \\
\hline F2 & & 1 & A & A & V & $\mathrm{O}$ & $\mathrm{O}$ & $\mathrm{O}$ & $\mathrm{V}$ & V & $\mathrm{O}$ & $\mathrm{V}$ & $\mathrm{O}$ & $\mathrm{O}$ \\
\hline F3 & & & 1 & $\mathrm{O}$ & $\mathrm{O}$ & $\mathrm{V}$ & A & $\mathrm{O}$ & $X$ & $\mathrm{~V}$ & V & $\mathrm{V}$ & $\mathrm{V}$ & $\mathrm{V}$ \\
\hline F4 & & & & 1 & $\mathrm{~V}$ & $\mathrm{~V}$ & A & $\mathrm{O}$ & $\mathrm{O}$ & V & $\mathrm{O}$ & $\mathrm{O}$ & V & $\mathrm{O}$ \\
\hline F5 & & & & & 1 & $\mathrm{~V}$ & $\mathrm{~A}$ & $\mathrm{~V}$ & A & A & V & $\mathrm{V}$ & A & $\mathrm{O}$ \\
\hline F6 & & & & & & 1 & A & A & A & A & V & A & A & $\mathrm{V}$ \\
\hline F7 & & & & & & & 1 & $X$ & $X$ & $\mathrm{~V}$ & V & $\mathrm{V}$ & $\mathrm{O}$ & $\mathrm{O}$ \\
\hline F8 & & & & & & & & 1 & $\mathrm{O}$ & $\mathrm{O}$ & V & $\mathrm{O}$ & $\mathrm{O}$ & $\mathrm{V}$ \\
\hline F9 & & & & & & & & & 1 & A & A & A & A & $\mathrm{V}$ \\
\hline F10 & & & & & & & & & & 1 & V & $\mathrm{V}$ & $\mathrm{V}$ & $\mathrm{V}$ \\
\hline F11 & & & & & & & & & & & 1 & $\mathrm{O}$ & A & A \\
\hline F12 & & & & & & & & & & & & 1 & $\mathrm{O}$ & $\mathrm{V}$ \\
\hline F13 & & & & & & & & & & & & & 1 & $\mathrm{~V}$ \\
\hline F14 & & & & & & & & & & & & & & 1 \\
\hline
\end{tabular}

Với nguyên lý đối xứng qua đường chéo (được thiết lập từ chuỗi số 1 xuất hiện trên Bảng 2), một bảng dữ liệu so sánh cặp yếu tố được thiết lập đầy đủ như Bảng 4, cụ thể:

+ Khi yếu tố F1 có giá trị quan hệ "V" với $\mathrm{F} 2$, theo chiều ngược lại thì F2 sẽ có mối quan hệ "A" với yếu tố F1,
+ Khi yếu tố F1 có giá trị quan hệ "A" với $\mathrm{F} 2$, theo chiều ngược lại thì $\mathrm{F} 2$ sẽ có mối quan hệ "V" với yếu tố F1,

+ Khi yếu tố $F 1$ có giá trị quan hệ " $\mathrm{X}$ " với $\mathrm{F} 2$, theo chiều ngược lại thì $\mathrm{F} 2$ sẽ có mối quan hệ " $X$ " với yếu tố $F 1$

+ Khi yếu tố $\mathrm{F} 1$ có giá trị quan hệ " $\mathrm{O}$ " với $\mathrm{F} 2$, theo chiều ngược lại thì $\mathrm{F} 2$ sẽ có mối quan hệ "A" với yếu tố F1.

Bảng 4. Bảng ma trận quan hệ so sánh cặp đầy đủ

\begin{tabular}{lllllllllllllll}
\hline & F1 & F2 & F3 & F4 & F5 & F6 & F7 & F8 & F9 & F10 & F11 & F12 & F13 & F14 \\
\hline F1 & I & A & V & O & V & V & O & O & V & V & V & V & V & V \\
F2 & V & 1 & A & A & V & O & O & O & V & V & O & V & O & O \\
F3 & A & V & 1 & O & O & V & A & O & X & V & V & V & V & V \\
F4 & O & V & O & 1 & V & V & A & O & O & V & O & O & V & O \\
F5 & A & A & O & A & 1 & V & A & V & A & A & V & V & A & O \\
F6 & A & O & A & A & A & 1 & A & A & A & A & V & A & A & V \\
F7 & O & O & V & V & V & V & 1 & X & X & V & V & V & O & O \\
F8 & O & O & O & O & A & V & X & 1 & O & O & V & O & O & V \\
F9 & A & A & X & O & V & V & X & O & 1 & A & A & A & A & V \\
F10 & A & A & A & A & V & V & A & O & V & 1 & V & V & V & V \\
F11 & A & O & A & O & A & A & A & A & V & A & 1 & O & A & A \\
F12 & A & A & A & O & A & V & A & O & V & A & O & 1 & O & V \\
F13 & A & O & A & A & V & V & O & O & V & A & V & O & 1 & V \\
F14 & A & O & A & O & O & A & O & A & A & A & V & A & A & 1 \\
\hline
\end{tabular}

- Bước 3: Xây dựng ma trận tiếp cận ban đầu dựa trên ma trận tương tác cấu trúc 
Với bước này, các kết quả "V, $\mathrm{A}, \mathrm{X}, \mathrm{O}$ " được hiển thị ở Bảng 5 được chuyển đổi thành hệ số nhị phân, tương ứng với các giá trị:
Nếu mối quan hệ được hiển thị mức "V" và "X" thì chuyển thành giá trị 1

Nếu mối quan hệ được hiển thị mức "A" và "O" thì chuyển thành giá trị 0 .

Bảng 5. Ma trận tiếp cận ban đầu dựa trên ma trận tương tác cấu trúc

\begin{tabular}{lcccccccccccccc}
\hline & F1 & F2 & F3 & F4 & F5 & F6 & F7 & F8 & F9 & F10 & F11 & F12 & F13 & F14 \\
\hline F1 & 1 & 0 & 1 & 0 & 1 & 1 & 0 & 0 & 1 & 1 & 1 & 1 & 1 & 1 \\
F2 & 1 & 1 & 0 & 0 & 1 & 0 & 0 & 0 & 1 & 1 & 0 & 1 & 0 & 0 \\
F3 & 0 & 1 & 1 & 0 & 0 & 1 & 0 & 0 & 1 & 1 & 1 & 1 & 1 & 1 \\
F4 & 0 & 1 & 0 & 1 & 1 & 1 & 0 & 0 & 0 & 1 & 0 & 0 & 1 & 0 \\
F5 & 0 & 0 & 0 & 0 & 1 & 1 & 0 & 1 & 0 & 0 & 1 & 1 & 0 & 0 \\
F6 & 0 & 0 & 0 & 0 & 0 & 1 & 0 & 0 & 0 & 0 & 1 & 0 & 0 & 1 \\
F7 & 0 & 0 & 1 & 1 & 1 & 1 & 1 & 1 & 1 & 1 & 1 & 1 & 0 & 0 \\
F8 & 0 & 0 & 0 & 0 & 0 & 1 & 1 & 1 & 0 & 0 & 1 & 0 & 0 & 1 \\
F9 & 0 & 0 & 1 & 0 & 1 & 1 & 1 & 0 & 1 & 0 & 0 & 0 & 0 & 1 \\
F10 & 0 & 0 & 0 & 0 & 1 & 1 & 0 & 0 & 1 & 1 & 1 & 1 & 1 & 1 \\
F11 & 0 & 0 & 0 & 0 & 0 & 0 & 0 & 0 & 1 & 0 & 1 & 0 & 0 & 0 \\
F12 & 0 & 0 & 0 & 0 & 0 & 1 & 0 & 0 & 1 & 0 & 0 & 1 & 0 & 1 \\
F13 & 0 & 0 & 0 & 0 & 1 & 1 & 0 & 0 & 1 & 0 & 1 & 0 & 1 & 1 \\
F14 & 0 & 0 & 0 & 0 & 0 & 0 & 0 & 0 & 0 & 0 & 1 & 0 & 0 & 1 \\
\hline
\end{tabular}

- Bước 4: Xây dựng ma trận tiếp cận cuối cùng.

Các giá trị "0" ở Bảng 4 được dịch chuyển sang "1" trong bước này nếu mối quan hệ giữa các yếu tố thể hiện được tính chất bắc cầu. Cụ thể, nếu tiêu chí $\mathrm{N} 2$ có liên quan đến tiêu chí N1 và yếu tố N1 có liên quan đến tiêu chí N3 thì tiêu chí N2 cũng liên quan đến tiêu chí N3. Khi đó, giá trị trong ô N2-N3 được chuyển từ 0 sang 1 , những ký hiệu là " $1 *$ " để phân biệt với mối quan hệ trực tiếp từ ma trận ban đầu được thiết lập. Tương tự, ta có các giá trị quan hệ bắc cầu còn lại, và được thể hiện ở Bảng 6 .

Bảng 6. Ma trận tiếp cận cuối cùng

\begin{tabular}{|c|c|c|c|c|c|c|c|c|c|c|c|c|c|c|c|}
\hline & F1 & F2 & F3 & F4 & F5 & F6 & F7 & F8 & F9 & F10 & F11 & F12 & F13 & F14 & DrP \\
\hline F1 & 1 & 0 & 1 & 0 & 1 & 1 & 0 & 0 & 1 & 1 & 1 & 1 & 1 & 1 & 10 \\
\hline F2 & 1 & 1 & $1 *$ & 0 & 1 & $1 *$ & $1 *$ & 0 & 1 & 1 & $1^{*}$ & 1 & $1 *$ & $1^{*}$ & 12 \\
\hline F3 & 0 & 1 & 1 & 0 & $1^{*}$ & 1 & $1 *$ & 0 & 1 & 1 & 1 & 1 & 1 & 1 & 11 \\
\hline F4 & 0 & 1 & 0 & 1 & 1 & 1 & 0 & $1 *$ & $1^{*}$ & 1 & $1^{*}$ & $1^{*}$ & 1 & $1 *$ & 11 \\
\hline F5 & 0 & 0 & 0 & 0 & 1 & 1 & $1 *$ & 1 & $1^{*}$ & 0 & 1 & 1 & 0 & $1 *$ & 8 \\
\hline F6 & 0 & 0 & 0 & 0 & 0 & 1 & 0 & 0 & $1^{*}$ & 0 & 1 & 0 & 0 & 1 & 4 \\
\hline F7 & 0 & $1^{*}$ & 1 & 1 & 1 & 1 & 1 & 1 & 1 & 1 & 1 & 1 & $1 *$ & $1 *$ & 13 \\
\hline F8 & 0 & 0 & $1^{*}$ & $1^{*}$ & $1 *$ & 1 & 1 & 1 & $1^{*}$ & $1 *$ & 1 & $1 *$ & 0 & 1 & 11 \\
\hline F9 & 0 & $1 *$ & 1 & $1 *$ & 1 & 1 & 1 & $1 *$ & 1 & $1 *$ & $1 *$ & $1^{*}$ & $1 *$ & 1 & 13 \\
\hline F10 & 0 & 0 & $1 *$ & 0 & 1 & 1 & $1 *$ & $1 *$ & 1 & 1 & 1 & 1 & 1 & 1 & 11 \\
\hline F11 & 0 & 0 & $1 *$ & 0 & $1^{*}$ & $1 *$ & $1 *$ & 0 & 1 & 0 & 1 & 0 & 0 & $1 *$ & 7 \\
\hline F12 & 0 & 0 & $1^{*}$ & 0 & $1^{*}$ & 1 & $1 *$ & 0 & 1 & 0 & $1 *$ & 1 & 0 & 1 & 8 \\
\hline F13 & 0 & 0 & $1 *$ & 0 & $1 *$ & 1 & 0 & $1^{*}$ & 1 & 0 & 1 & $1 *$ & 1 & 1 & 9 \\
\hline F14 & 0 & 0 & 0 & 0 & 0 & 0 & 0 & 0 & 0 & 0 & 1 & 0 & 0 & 1 & 2 \\
\hline DP & 2 & 5 & 10 & 4 & 12 & 13 & 9 & 7 & 13 & 8 & 14 & 11 & 8 & 14 & \\
\hline
\end{tabular}

1 *: Mối quan hệ mang tính chất bắc cầu 
- Bước 5: Phân cấp các yếu tố
Từ ma trận tiếp cận cuối cùng, các yếu tố quan hệ theo hàng và quan hệ theo cột được xác định (Bảng 7).

Bảng 7. Phân cấp các yếu tố

\begin{tabular}{|c|c|c|c|c|}
\hline Nhân tố & Tập hợp đạt được & Tập hợp giúp đạt được & Tập hợp giao & Cấp \\
\hline F1 & $1,3,5,6,9,10,11,12,13,14$ & 1,2 & 1 & IV \\
\hline F2 & $1,2,3,5,6,7,9,10,11,12,13,14$ & $2,3,4,7,9$ & $2,3,7,9$ & $\mathrm{~V}$ \\
\hline F3 & $2,3,5,6,7,9,10,11,12,13,14$ & $1,2,3,7,8,9,10,11,12,13$ & $2,3,7,9,10,11,12$ & $\mathrm{~V}$ \\
\hline F4 & $2,4,5,6,8,9,10,11,12,13,14$ & $4,7,8,9$ & $4,8,9$ & $\mathrm{~V}$ \\
\hline F5 & $5,6,7,8,9,11,12,14$ & $\begin{array}{l}1,2,3,4,5,7,8,9,10,11,12, \\
13\end{array}$ & $5,7,8,9,11,12$ & III \\
\hline F6 & $6,9,11,14$ & $\begin{array}{l}1,2,3,4,5,6,7,8,9,10,11, \\
12,13\end{array}$ & $6,9,11$ & II \\
\hline F7 & $\begin{array}{l}2,3,4,5,6,7,8,9,10,11,12,13 \\
14\end{array}$ & $2,3,5,7,8,9,10,11,12$ & $2,3,5,7,8,9,10,11,12$ & VI \\
\hline F8 & $3,4,5,6,7,8,9,10,11,12,14$ & $4,5,7,8,9,10,13$ & $4,5,7,8,9,10$ & $\mathrm{~V}$ \\
\hline F9 & $\begin{array}{l}2,3,4,5,6,7,8,9,10,11,12,13, \\
14\end{array}$ & $\begin{array}{l}1,2,3,4,5,6,7,8,9,10,11, \\
12,13\end{array}$ & $\begin{array}{l}2,3,4,5,6,7,8,9,10 \\
11,12,13\end{array}$ & \\
\hline F10 & $3,5,6,7,8,9,10,11,12,13,14$ & $1,2,3,4,7,8,9,10$ & $3,7,8,9,10$ & $\mathrm{~V}$ \\
\hline F11 & $3,5,6,7,9,11,14$ & $\begin{array}{l}1,2,3,4,5,6,7,8,9,10,11, \\
12,13,14\end{array}$ & $3,5,6,7,9,11,14$ & II \\
\hline F12 & $3,5,6,7,9,11,12,14$ & $1,2,3,4,5,7,8,9,10,12,13$ & $3,5,7,9,12$ & III \\
\hline F13 & $3,5,6,8,9,11,12,13,14$ & $1,2,3,4,7,9,10,13$ & $3,9,13$ & IV \\
\hline F14 & 11,14 & $\begin{array}{l}1,2,3,4,5,6,7,8,9,10,11, \\
12,13,14\end{array}$ & 11,14 & I \\
\hline
\end{tabular}

- Bước 6: Hình thành mô hình ISM

Từ kết quả phân cấp được hiển thị ở Bảng 7 , sơ đồ cấu trúc được hình thành với sự kết hợp xem xét mối quan hệ từ Bảng 5 , một sơ đồ thể hiện mối quan hệ theo cấp bậc được hình thành với đầy đủ mối quan hệ giữa các yếu tố, hỗ trợ cho quá trình đánh giá mức độ quan trọng của các yếu tố ở giai đoạn tiếp theo, được hiển thị ở Hình 5.

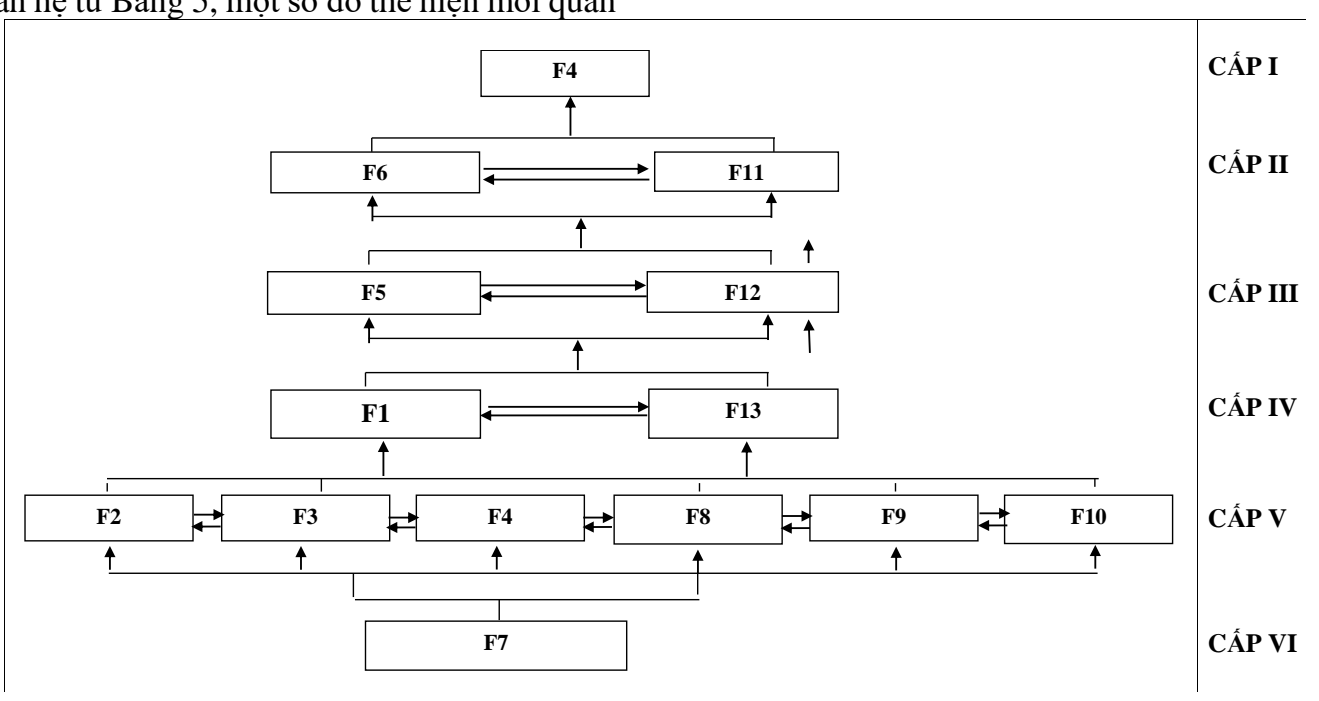

Hình 5. Mối quan hệ cấp bậc trong mô hình ISM

- Sử dụng phương pháp MICMAC 
Từ kết quả của phương pháp ISM, hình thành các cụm bằng phương pháp MICMAC. Để phân chia được tiêu chí vào các cụm, tiến hành tính các giá trị hàng và cột dựa trên ma trận tiếp cận cuối cùng. Trong đó, mức độ định hướng $(\mathrm{DrP})$ và mức độ phụ thuộc (DP) được hiển thị ở Bảng 8 .

Bảng 8. Mức độ định hướng và phụ thuộc của từng yếu tố

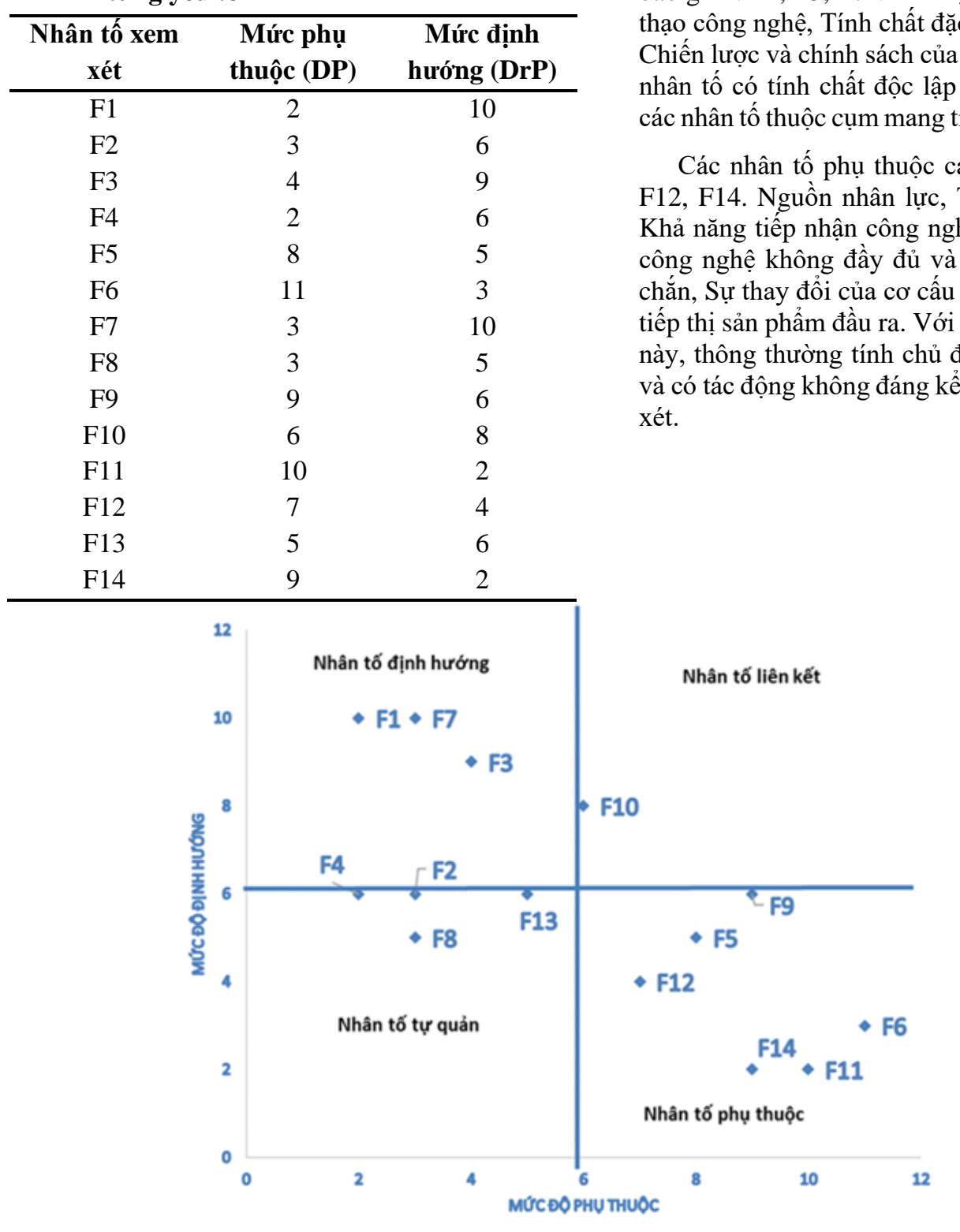

Hình 6. Ma trận tác động chéo theo phương pháp MICMAC

Các nhân tố thuộc cụm tự quản bao gồm: F2, F4, F8 và F13 (Mức độ phức tạp của công nghệ, Tài chính của doanh nghiệp, Cơ chế chuyển giao, và Vốn sản xuất) và các nhân tố thuộc cụm liên kết bao
Từ kết quả Bảng 8 , một ma trận tác động chéo áp dụng cho phân loại được thiết lập dựa trên nguyên lý đồ thị, với trục tung tương ứng với mức độ định hướng (DrP) và mức độ phụ thuộc (DP) được diễn đạt thông qua trục hoành, cụ thể được hiển thị ở Hình 6.

Từ Hình 6 , các nhân tố thuộc cụm định hướng bao gồm: F1, F3, F7 với tên gọi là Khả năng thông thạo công nghệ, Tính chất đặc thù doanh nghiệp, và Chiến lược và chính sách của Chính phủ. Đây là các nhân tố có tính chất độc lập cao, giúp định hướng 列

cao là F5, F6, F9, F11, có tác động không đáng kể đến kết quả được xem xét.

gồm: F10 (Mức độ ưu tiên của dự án). Đây là các nhân tố mang tính chất trung gian trong việc tạo cầu nối giữa cụm độc lập và cụm phụ thuộc. Mặc dù tính độc lập đã bị tác động đáng kể bởi tính phụ thuộc, 
nhưng các nhân tố thuộc 2 cụm này vẫn có một mức độ quan trọng nhất định, tác động đến kết quả cuối cùng. Đặc biệt, trong nghiên cứu này, các nhân tố thuộc 2 cụm này có sự khác biệt không đáng kể để có thể xem xét chúng vẫn nằm trong cụm định hướng.

Từ kết quả của phương pháp ISM - MICMAC, mức độ quan trọng và mức độ về mối quan hệ giữa các nhân tố và các cụm nhân tố được xác định. Các nhân tố thuộc cụm định hướng và lân cận tạo ra các tác động lớn đến khả năng chuyển giao, ứng dụng, đổi mới công nghệ trên địa bàn TP. Cần Thơ. Các nhân tố bao gồm: F1, F2, F3, F4, F7, F10, F13 hay cụ thể là Khả năng thông thạo công nghệ, Mức độ phức tạp của công nghệ, Tính chất đặc thù doanh nghiệp, Tài chính của doanh nghiệp, Chiến lược và chính sách của Chính phủ, Mức độ ưu tiên của dự án và Vốn sản xuất. Các nhân tố này có vai trò đặc biệt quan trọng trong việc đề xuất xây dựng các giải pháp để thúc đẩy hoạt động chuyển giao, ứng dụng, đổi mới công nghệ trên địa bàn TP. Cần Thơ trong tương lai sắp tới.

\section{KẾT LUẬN VÀ ĐỀ XUẤT}

\subsection{Kết luận}

Nghiên cứu nhằm xác định các yếu tố chính tác động đến hoạt động chuyển giao, ứng dụng, đổi mới công nghệ tại TP. Cần Thơ. Mô hình ISM và MICMAC được sử dụng bằng cách phân tích thống kê các kết quả khảo sát và tham vấn ý kiến từ 217 doanh nghiệp trên địa bàn TP. Cần Thơ và nhiều chuyên gia về khoa học và công nghệ của khu vực ĐBSCL và Việt Nam, có sự thống nhất rất tốt với dữ liệu thử nghiệm, nghiên cứu cho thấy các yếu tố trong cụm "Nhân tố định hướng", bao gồm Khả năng thông thạo công nghệ, Tính chất đặc thù doanh nghiệp và Chiến lược và chính sách của Chính phủ có tác động mạnh mẽ nhất đến hoạt động này. Bên cạnh đó, các yếu tố Mức độ phức tạp của công nghệ, Tài chính của doanh nghiệp, Mức độ ưu tiên của dự án, và Vốn sản xuất cũng có mức độ quan trọng nhất định, không được bỏ qua và cần được chú trọng nhiều không kém các yếu tố trong cụm "Nhân tố định hướng". Kết quả thu được từ bài báo này tạo thành một cơ sở lý luận cho các giải pháp được xây dựng trong việc quản lý công nghệ của doanh nghiệp cũng như của các cấp chính quyền. Kết quả từ bài báo tạo cơ sở vững chắc hơn trong việc đưa ra gợi ý về hàm ý chính sách để thúc đẩy hoạt động chuyển giao, ứng dụng, đổi mới công nghệ tại TP. Cần Thơ.

\section{2. Đề xuất}

Kết quả của nghiên cứu cho thấy Khả năng thông thạo công nghệ, Mức độ phức tạp của công nghệ, Tính chất đặc thù doanh nghiệp, Tài chính của doanh nghiệp, Chiến lược và chính sách của Chính phủ, Mức độ ưu tiên của dự án và Vốn sản xuất có vai trò đặc biệt quan trọng. Do đó, các cấp quản lý và các doanh nghiệp trên địa bàn TP. Cần Thơ nên có tiếng nói chung trong việc xây dựng chiến lược hoạt động của mình. Trước mắt, một cơ chế phân cấp chặt chẽ là cần thiết để tạo cơ sở pháp lý trong việc huy động nguồn vốn và các nguồn hỗ trợ cho doanh nghiệp liên quan đến hoạt động về công nghệ trên địa bàn, tiếp theo đó là một bước chuẩn bị về nhân lực công nghệ, tập trung vào các trường đại học làm cầu nối.

\section{LỜI CẢM TẠ}

Bài báo này là sản phẩm của đề tài nghiên cứu khoa học xã hội và nhân văn cấp TP. Cần Thơ với mã số 19/HĐ-SKHCN.

Nghiên cứu sinh Nguyễn Thắng Lợi được tài trợ bởi Tập đoàn Vingroup và hỗ trợ bởi chương trình học bổng đào tạo thạc sĩ, tiến sĩ trong nước của Quỹ Đổi mới sáng tạo Vingroup (VINIF), Viện Nghiên cứu Dữ liệu lớn (VinBigdata), mã số VINIF.2020.TS.26

\section{TÀI LIỆ THAM KHẢO}

Ahmed, MT., Saleh, M., \& Abdelrehim, A. (2009). El Maghara Scenario A search for Sustainability and Equity: An Egyption Case Study. Journal of Futures Studies 14(2), 55-89.

Cancino, C. A., Paza, A. I. L., Ramaprasad, A., \& Syn, T. (2018). Technological innovation for sustainable growth: An ontological perspective. Journal of Cleaner Production, 179, 31-41.

Ejdys, J., Matuszak-Flejszman, A., Szymanski, M., Ustinovicius, L., Shevchenko, G., \& LulewiczSas, A. (2016). Crucial factors for Improving the ISO14001 Environmental Management System. Journal of Business Economics and Management, 17(1), 52-73.

Godet, M., \& Durance, P. (2008). La Prospective stratégique, pour les entreprises et les territoires. Paris: Dunod.

Guo, Y., Xia, X., Zhang, S., \& Zhang, D. (2018). Environmental regulation, government R\&D funding and green technology innovation: Evidence from China provincial data. Sustainability, 10(4), 940, 1-21.

Halicka, K. (2016). Prospektywna analiza technologii - metodologia i procedury badawcze [Prospective analysis of technology - 
methodology and test procedures]. Białystok: Oficyna Wydawnicza Politechniki Białostockiej.

Jugend, D., Jabbour, C. J. C., Scaliza, J. A. A., Rocha, R. S., Junior, J. A. G., ... Salgado, M. H. (2018). Relationships among open innovation, innovative performance, government support and firm size: Comparing Brazilian firms embracing different levels of radicalism in innovation. Technovation, 74-75, 54-65.

Klooster, SA., \& Asselt, MBA. (2006). Practising the scenario-axes technique. Futures, 38, 15-30.

Luật Chuyển giao công nghệ số 07/2017/QH14, Luật chuyển giao công nghệ sửa đổi đã chính thức được thông qua vào ngày 19/6/2017.

Morel-Guimaraes, L., Khalil, T. M., Hosni, Y. A. (2005). Management of Technology: Key Success Factors for Innovation and Sustainable Development: Selected Papers from the Twelfth International Conference on Management of Technology. Published by Elsevier Science.

Nazarko, J., \& Kononiuk, A. (2013). The critical analysis of scenario construction in the Polish foresight initiatives. Technological and Economic Development of Economy, 19(3), 510-532.

Ngọc Thảo. (2021). Công nghiệp Cần Thơ: Động lực tăng trưởng vùng Đồng bằng sông Cửu Long.
Báo Công Thuoong Online ngày 28/04/2021 tại: https://congthuong.vn/cong-nghiep-can-thodong-luc-tang-truong-vung-dong-bang-song-cuulong-156077.html

Nguyễn Thị Thơm. (2020). Thúc đẩy chuyển giao và đổi mới công nghệ. Bài đăng trên Tạp chí Lý luận chính trị số 8-2020 tại: https://www.most.gov.vn/vn/tin-tuc/19565/thucday-chuyen-giao-va-doi-moi-cong-nghe.aspx.

Quyết định số 118/QĐ-TTg của Thủ tướng Chính phủ ngày 25/01/2021: Ban hành Chương trình đổi mới công nghệ quốc gia đến năm 2030,

Slovin, M. B., \& Sushka, M. E. (1984). A note on the evidence on alternative models of the banking firm: A cross section study of commercial loan rates. Journal of Banking \& Finance, 8(1), 99-108.

Warfield, J. N. (1973). Binary Matrices in System Modeling. Transactions on Systems, 3, 441- 449.

Yang, Z., Linxiu, W., \& Fanbo, X., (2014). The Analysis of High-tech Industrial Development Zone Investment Environment on the Basis of ISM -Taking Xuzhou High-tech Industrial Development Zone as a Case. Science and Technology Management Research 2014, (3), 127-130. 